\title{
Observation of Quantum Spin Noise in a 1D Light-Atoms Quantum Interface
}

\author{
J.-B. Béguin, ${ }^{*}$ J. H. Müller, J. Appel, ${ }^{\dagger}$ and E. S. Polzik \\ QUANTOP, Niels Bohr Institute, University of Copenhagen, Blegdamsvej 17, 2100 Copenhagen, Denmark
}

(Received 1 February 2018; revised manuscript received 14 June 2018; published 12 July 2018)

\begin{abstract}
We observe collective quantum spin states of an ensemble of atoms in a one-dimensional light-atom interface. Strings of hundreds of cesium atoms trapped in the evanescent field of a tapered nanofiber are prepared in a coherent spin state, a superposition of the two clock states. A weak quantum nondemolition measurement of one projection of the collective spin is performed using a detuned probe dispersively coupled to the collective atomic observable, followed by a strong destructive measurement of the same spin projection. For the coherent spin state we achieve the value of the quantum projection noise $40 \mathrm{~dB}$ above the detection noise without atoms, well above the $3 \mathrm{~dB}$ required for reconstruction of the negative Wigner function of nonclassical states. We analyze the effects of strong spatial inhomogeneity inherent to atoms trapped and probed by the evanescent waves. We furthermore study temporal dynamics of quantum fluctuations relevant for measurement-induced spin squeezing and assess the impact of thermal atomic motion. This work paves the road towards observation of spin-squeezed and entangled states and manybody interactions in $1 \mathrm{D}$ spin ensembles.
\end{abstract}

DOI: 10.1103/PhysRevX.8.031010

\section{INTRODUCTION}

Quantum superpositions, squeezed states, and multipartite entanglement are features of the quantum world which are central for the future developments of quantum information science and metrology. These fundamental nonclassical phenomena have been extensively studied and exploited with an efficient quantum interface between light and ensembles of atoms [1], offering an exciting alternative to cavity quantum electrodynamics with single particles.

One of the most recent and promising examples of such light-atom interface uses an optical nanofiber. Its essence is in combining guiding of light and trapping of cold atoms in the subwavelength evanescent field of a tapered fiber. This gives rise to strong light-atom interactions in a genuine onedimensional geometry [2-5].

Among the recent successes of this emerging platform are generation of highly sub-Poissonian atom number distributions [4], realization of electromagnetically induced transparency-based memories [6,7], achieving the strong

\footnotetext{
*Norman Bridge Laboratory of Physics 12-33, California Institute of Technology, Pasadena, CA 91125, USA.

jappel@nbi.dk

polzik@nbi.dk

Published by the American Physical Society under the terms of the Creative Commons Attribution 4.0 International license. Further distribution of this work must maintain attribution to the author(s) and the published article's title, journal citation, and DOI.
}

Subject Areas: Atomic and Molecular Physics, Quantum Physics
cavity-QED regime for a single atom [5], and demonstration of nano-optical mirrors with a few structured atoms $[8,9]$. These new functionalities become enabling tools to implement exciting proposals for studies of one-dimensional many-body effects [10]. The intrinsic fiber compatibility of this interface makes it particularly attractive for scalable quantum fiber networks.

In order to open the realm of continuous variable information processing and quantum metrology with nanofiber interfaces, two well-recognized objectives have to be pursued. The first one is the ability to characterize the preparation of collective quantum states of atoms with high-precision tomography $[11,12]$. The second challenging goal is the realization of a quantum nondemolition (QND) measurement on collective atomic states, which is one way for creation of squeezed states $[13,14]$, with applications to entanglement-assisted atomic clocks and magnetometers as well as a component in quantum teleportation and memory protocols [1].

In this work, for the first time, we observe quantum fluctuations of the internal atomic state in a nanofiber trapped atomic ensemble and present an optimized dispersive probing scheme giving unprecedented signal-to-noise ratio (SNR). We perform quantum state tomography using a QND measurement in a nanofiber trapped atomic ensemble at the projection noise level. We present a framework for QND measurements in inhomogeneously coupled ensembles. Our findings indicate that thermal motion and mechanical backaction forces are very relevant and that cooling of the atomic motion is a necessary prerequisite for further 
advancement of quantum control in general in nano-optical light-atom interfaces.

The paper is structured as follows. First, we explain how we measure the atomic populations in two electronic quantum states precisely. For an ensemble prepared in a quantum superposition state, we then demonstrate that the fluctuations of the population difference originate from quantum projection noise. Finally, we present a framework for investigating the effect of atomic motion on preparing a spin-squeezed state using a weak QND measurement in a strongly inhomogeneously coupled ensemble.

\section{ATOMIC POPULATION MEASUREMENT}

The experiment begins with loading the nanofiber trapping sites [Fig. 1(a)] with cold atoms [4] from a magneto-optical trap (MOT) and preparing them in the lower clock level $|3,0\rangle \equiv\left(6^{2} S_{1 / 2}, F=3, m_{F}=0\right)$ [Fig. 1(c)] as follows.

We first accumulate the majority of the atoms in the upper clock level $|4,0\rangle \equiv\left(6{ }^{2} S_{1 / 2}, F=4, m_{F}=0\right)$ using

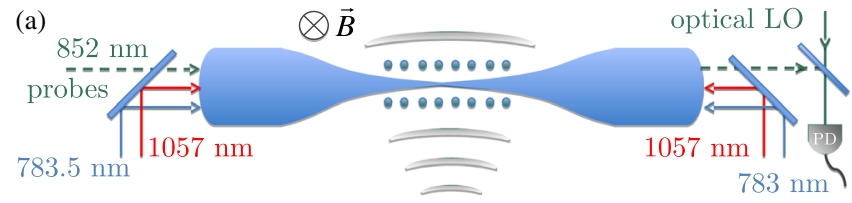

(b)
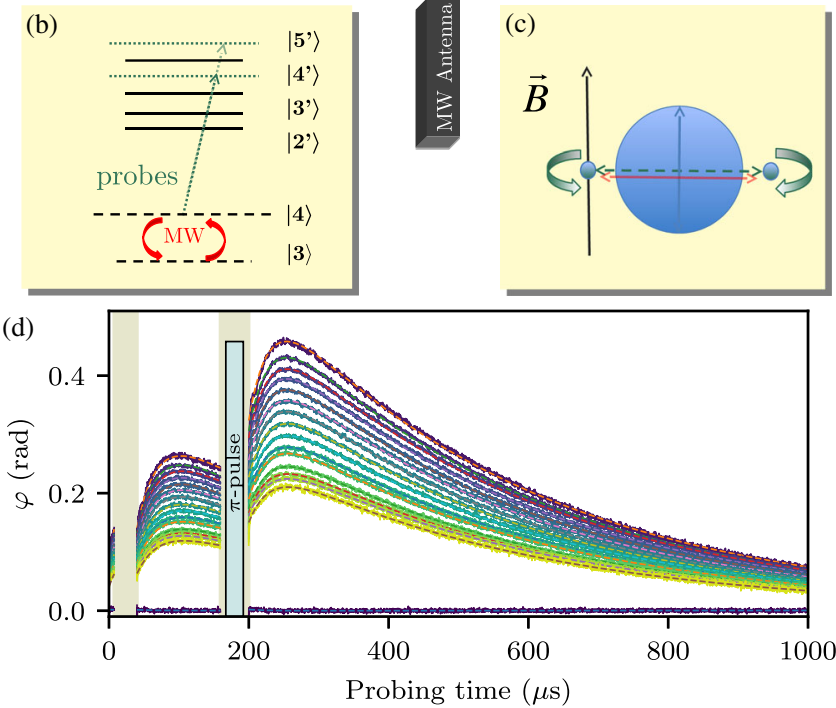

FIG. 1. (a) Sketch of nanofiber trap setup with light fields for trapping and probing and microwave source for coherent transfer between clock levels (MW: Microwave; PD: Photo detector). (b) Relevant atomic energy levels for cesium. (c) Transverse section of nanofiber with atomic trap sites, local probe light polarization, and magnetic bias field direction indicated. (d) Dispersive detection of the clock state populations for an increasing number (bottom to top) of atoms prepared in the superposition state $|\Psi\rangle$. Solid lines are averages over typically 300 realizations. Dashed lines are fits to the model presented in Eq. (2). See main text for state preparation and measurement procedure; shaded areas indicate interruption of the probing. dark state optical pumping on the $|4\rangle \equiv\left(6^{2} S_{1 / 2}, F=4\right) \rightarrow$ $\left|4^{\prime}\right\rangle \equiv\left(6^{2} P_{3 / 2}, F=4\right)$ transition with $\pi$-polarized light propagating through the nanofiber and external repumping light on $|3\rangle \equiv\left(6^{2} S_{1 / 2}, F=3\right) \rightarrow\left|4^{\prime}\right\rangle$. A magnetic bias field of $3 \mathrm{G}$ applied along the polarization direction of the blue trap light defines the quantization axis [Fig. 1(c)]. Using a resonant microwave Rabi $\pi$ pulse, we transfer the accumulated atoms into $|3,0\rangle$ before pushing out of the trap any atoms remaining in level $|4\rangle$ using external blue detuned and circularly polarized light on the $|4\rangle \rightarrow$ $\left(6^{2} P_{3 / 2}, F=5\right) \equiv\left|5^{\prime}\right\rangle$ transition. Finally, we are left with a pure spin-polarized ensemble of up to $N_{\text {at }} \sim 1500$ atoms in $|3,0\rangle$, corresponding to an overall pumping efficiency of about $65 \%$, similar to values reported in Ref. [15]. Using a resonant $\pi / 2$ microwave pulse we prepare the ensemble of atoms in the superposition of the clock states, $|\Psi\rangle=\stackrel{N_{\text {at }}}{\otimes}$ $(|3,0\rangle+|4,0\rangle) / \sqrt{2}$.

A prerequisite to the characterization of collective atomic states is a measurement method of the atomic basis state populations. To this end, building on the versatile and minimally invasive dual-color dispersive probing method developed in Ref. [4], we measure the atomic populations in $|3,0\rangle$ and $|4,0\rangle$.

Two probe light frequencies, roughly symmetrically red and blue detuned by $62.5 \mathrm{MHz}$ with respect to the $|4\rangle \rightarrow$ $\left|5^{\prime}\right\rangle$ transition, are sent through the nanofiber [Fig. 1(b)]. The differential phase shift $\vec{\varphi}(t)$ [16] of these fields due to off-resonant interaction with atoms in $F=4$ is detected via heterodyne detection with an external optical local oscillator tuned exactly in the middle between the probe frequencies. This allows for a shot-noise-limited dispersive detection of $F=4$ atoms with an overall homodyne detection efficiency $\kappa=0.4$ [17].

Using Ramsey spectroscopy, we carefully chose the precise probe detuning to eliminate the probe-induced differential light shift between $|4,0\rangle$ and $|3,0\rangle$ so that the mean mechanical effect of probe light onto atoms in these states vanishes as well.

The probe field polarization is aligned parallel to the red-detuned trapping light. Because of the in-quadrature longitudinal field component of the evanescent probe mode at the location of the atomic trap potential minima, the local polarization is almost circular $\left(92 \% \sigma^{ \pm}\right.$and $\left.8 \% \sigma^{\mp}\right)$ with opposite helicity for the two 1D ensembles of atoms trapped on either side along the nanofiber $[18,19]$. Consequently, during probing, atoms initially prepared in the state $|4,0\rangle$ will be pumped progressively into the extreme Zeeman levels $|4, \pm 4\rangle$. Here, the probe interaction occurs predominantly via the strong and closed $|4, \pm 4\rangle \leftrightarrow$ $|5, \pm 5\rangle$ transition, which increases the interaction strength and reduces decays to the $F=3$ hyperfine level.

The dynamics of the evolution of the mean differential phase shift $\langle\vec{\varphi}(t)\rangle=\varphi_{0} \cdot \vec{m}(t)$ caused by atoms initially in $|4,0\rangle$ is well described by a phenomenological model, 


$$
\vec{m}_{\text {model }}(t)=\left(\beta-(\beta-1) e^{-t / \tau_{\mathrm{at}}}\right) e^{-t / \tau_{\text {loss }}},
$$

where the time constant $\tau_{\text {at }}$ describes the speed of pumping towards the extreme Zeeman states, $\varphi_{0}$ denotes the initial phase shift at $t=0$, and $\beta$ models the increase in interaction strength during Zeeman pumping. The time constant $\tau_{\text {loss }}$ is associated with random loss of atomic population out of $|4\rangle$, caused predominantly by probe absorption induced heating and to a much smaller extent by pumping into the $F=3$ hyperfine manifold.

By fitting Eq. (1) to traces recorded with a varying number of atoms $N_{4}$ initially in $|4,0\rangle$, we verify experimentally that $\tau_{\text {at }}, \tau_{\text {loss }}$, and $\beta$ are independent of the total number of atoms and that they can be calibrated for fixed experimental conditions (trap and probe powers) such that $\varphi_{0}$ is proportional to the number of atoms: $\varphi_{0}=N_{4} \varphi_{\text {eff }, 1}$.

To determine the population $N_{4}$ in the $|4,0\rangle$ and the population $N_{3}$ in $|3,0\rangle$ in a single realization of a quantum state, we use the following measurement sequence. First, we probe the differential phase shift $\varphi(t)$ caused by the atoms in $F=4$ for a time $\sim 6 \tau_{\text {at }}$ (with a $32 \mu$ s interruption, see QND measurements: weak probing and temporal dynamics). After this time, all atoms are pumped out of $|4,0\rangle$ and accumulated in the $|4, \pm 4\rangle$ states. Then, we briefly turn off the probe light for $40 \mu \mathrm{s}$, apply a microwave $\pi$ pulse to transfer the population of $|3,0\rangle$ into $|4,0\rangle$, and again probe the ensemble for $\sim 20 \tau_{\text {at }}$ [see Fig. 1(d)].

We concatenate these successive measurements into a single trace, and the temporal dynamics of the recorded traces are well described by

$$
\vec{\varphi}(t)=\left\{\begin{array}{lc}
\varphi_{4} \cdot \vec{m}(t) & 0 \leq t<t_{\text {flip }} \\
\varphi_{4} \cdot \vec{m}(t)+\varphi_{3} \cdot \vec{m}\left(t-t_{\text {flip }}\right) & t_{\text {flip }} \leq t
\end{array}\right.
$$

A linear least squares fit to the measured data yields $\varphi_{4}$ and $\varphi_{3}$, where $\varphi_{4}=\varphi_{\text {eff }, 1} N_{4}$ and $\varphi_{3}=\varphi_{\text {eff, } 1} N_{3} . \varphi_{\text {eff, } 1}$ denotes the effective differential phase shift caused by a single atom in the state $|4,0\rangle$, and $N_{3}$ and $N_{4}$ are the initial atomic populations in the $|3,0\rangle$ and $|4,0\rangle$ clock levels. $t_{\text {flip }}$ indicates the beginning of the $|3,0\rangle$ population measurement.

$\varphi_{\text {eff, }, 1}$ is directly proportional to the single-atom optical depth, and to establish an absolute atom number, it has to either be calculated from first principles, measured separately, or can be deduced from the scaling of the projection noise, as outlined in the next section (see also $N_{\text {at }}$ versus $N_{\text {eff }}$ discrepancy).

\section{PROJECTION NOISE}

In the following we use this technique to demonstrate, for the first time, the measurement of quantum fluctuations of ensembles coupled to a single-mode waveguide.

Again, we start by preparing our ensemble in the coherent spin state (CSS) $|\Psi\rangle$. With microwave Ramsey spectroscopy we observe a bare coherence time $\mathcal{T}_{2}$ of a few hundreds of microseconds, that can be increased with spin echo to a few milliseconds, in agreement with the observations of Reitz et al. [15].

In order to observe the fundamental quantum fluctuations of the atomic populations of this coherent superposition state, it is necessary to reject the shot-to-shot fluctuations in the number of atoms inherent to state preparation (e.g., loading noise); i.e., we need to know the number of atoms initially in $|4,0\rangle$ and $|3,0\rangle$.

Knowing $\varphi_{4}$ and $\varphi_{3}$, for each single-shot measurement trace according to Eq. (2), we define the phase estimator proportional to the total atom number $N_{\text {at }}=N_{4}+N_{3}$ as $\varphi_{N}=\varphi_{4}+\varphi_{3}$ and for the population difference $\Delta N=$ $N_{4}-N_{3}$ as $\varphi_{\Delta}=\varphi_{4}-\varphi_{3}$. For a CSS the latter should be subject to the projection noise: For a CSS the variances of the populations are $\operatorname{var}\left(N_{4}\right)=\operatorname{var}\left(N_{3}\right)=\left\langle N_{\text {at }}\right\rangle / 4$ and $\operatorname{var}(\Delta N)=\left\langle N_{\text {at }}\right\rangle$. From this we see that for the projectionnoise-limited state $\operatorname{var}\left(\varphi_{\Delta}\right)-\delta \varphi_{\mathrm{sn}}{ }^{2}=\varphi_{\text {eff }, 1}{ }^{2} N_{\text {at }}=\varphi_{\text {eff }, 1}\left\langle\varphi_{N}\right\rangle$ scales linearly with $\left\langle\varphi_{N}\right\rangle$. Here, $\delta \varphi_{\mathrm{sn}}{ }^{2}$ denotes measurement phase noise in the absence of atoms such as shot noise and detector electronic noise. In Fig. 2, we present $\operatorname{var}\left(\varphi_{\Delta}\right)$ as a function of $\left\langle\varphi_{N}\right\rangle$. Each point corresponds to a bin of around 200 experiments with a similar atom number. The scaling is linear with a negligible quadratic part over a wide range of atom numbers. For the experimental realizations with the maximum atom number, $\operatorname{var}\left(\varphi_{\Delta}\right)$ reaches up to $40 \mathrm{~dB}$ above its value in the absence of atoms $\delta \varphi_{\mathrm{sn}}{ }^{2}$, i.e., above the detection noise alone. From the slope of a linear fit one can extract an effective phase shift per atom and an effective

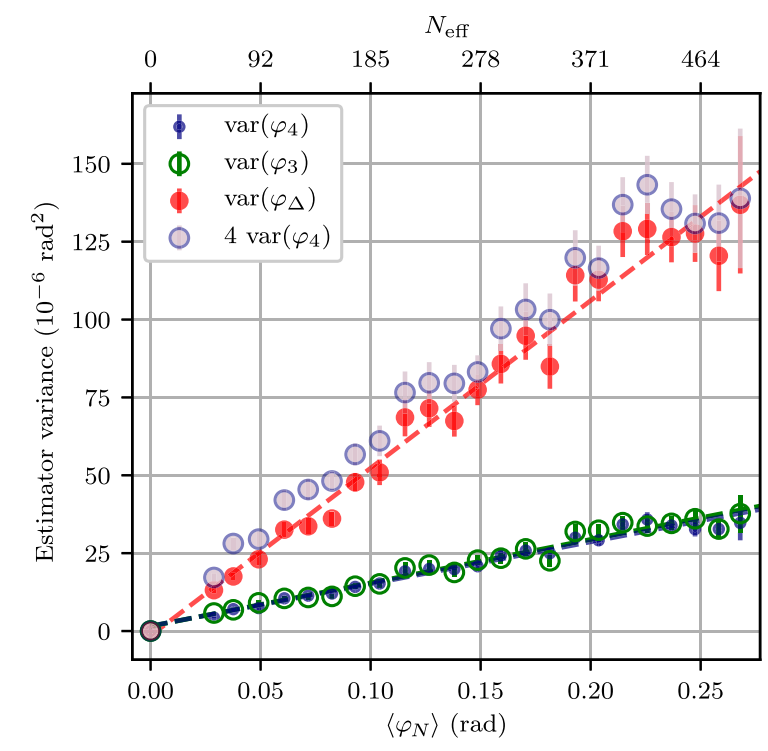

FIG. 2. Noise scaling of the atomic population estimators. Green and blue symbols are variances of $\varphi_{4}$ and $\varphi_{3}$, red filled circles show the variance of $\varphi_{\Delta}$, rose circles show variance of $\varphi_{4}$ scaled by a factor of 4 . Error bars are statistical, dashed lines are linear fits to the data of same color. The top $x$ axis shows the inferred effective atom number $N_{\text {eff }}$. Data were taken under similar conditions as in Fig. 1(d). 
atom number using the relation $d \operatorname{var}\left(\varphi_{\Delta}\right) / d\left\langle\varphi_{N}\right\rangle=\varphi_{\text {eff,1 }}$ with $\varphi_{N}=\varphi_{\text {eff }, 1} N_{\text {eff }}$. For atoms in $|4,4\rangle$ the resulting phase shift per atom is $\beta \varphi_{\text {eff }, 1}=2 \mathrm{mrad}$, corresponding to a single-atom optical depth of $\operatorname{od}_{1}=9 \%$ on the cycling transition) [20,21]. In addition to the negligible quadratic component which characterizes atom-number-dependent technical noise [22], the observed ratio of the slopes in Fig. 2, $\operatorname{var}\left(\varphi_{4}\right) / \operatorname{var}\left(\varphi_{\Delta}\right) \simeq 4$, presents a strong confirmation of the quantum projection noise nature of the presented data, as it is consistent with almost purely anticorrelated fluctuations of the two clock levels. We find negligible additional noise when replacing $\pi / 2$ pulses by $3 \pi / 2$ pulses, which rules out microwave power fluctuations as its origin.

\section{A. $N_{\text {at }}$ versus $N_{\text {eff }}$ discrepancy}

We can compare $N_{\text {eff }}$ extracted from the projection noise scaling to the atom number $N_{\text {at }}$ obtained via an independent method based on recording optical pumping transients [4] which is robust against important systematic effects such as inhomogeneous coupling to the probe light. We observe a systematic difference $\Upsilon=N_{\text {at }} / N_{\text {eff }}=2$, a discrepancy similar to those reported for various other interfaces, and treat the effective atom number as in Ref. [20].

We attribute this effect to the coupling inhomogeneity amongst the trapped thermal atoms located at different positions within the radially decaying probe field. Such inhomogeneous coupling results in $\Upsilon=1+\operatorname{var}\left(\overline{\varphi_{\text {eff }, 1}}\right) /$ $\left\langle\overline{\varphi_{\text {eff }, 1}}\right\rangle^{2}>1$, where the overscore denotes temporal averaging over the probing time; variance and mean are taken over the atoms of the ensemble. Because of the exponential tail of the conservative trapping potential, even with relatively long averaging times of many tens of radial trap oscillation periods, the mean coupling strength of the individual atoms still differs. Numerical simulations of the classical trajectories for atoms with temperatures above $90 \mu \mathrm{K}$ consistently result in $\Upsilon>1.5$, even for averaging times beyond $150 \mu \mathrm{s}$.

\section{QND MEASUREMENTS: WEAK PROBING AND TEMPORAL DYNAMICS}

In the following, we present a framework for performing weak (i.e., nonprojective) measurements in the presence of thermal motion, inhomogeneous coupling, and partition noise. We elaborate on how to choose the temporal modes of detection to extract the optimal amount of information for spin squeezing or metrology by QND measurements.

For an ensemble-waveguide quantum interface of $N_{\text {at }}$ atoms, ideally a single optical mode should interface with a single mode of the $N_{\text {at }}$ orthogonal atomic modes.

By definition, in nano-optical waveguide structures, electrical fields vary spatially on the optical wavelength scale, so that inhomogeneous coupling of the individual quantum systems to the guided light mode is omnipresent.
Motion of atoms within an inhomogeneous probe field limits the performance for implementing a quantum interface, since it introduces temporal dependence on the atomic mode that the optical mode couples to: information written into such an ensemble cannot be retrieved perfectly when the atomic modes during the write-and-read process overlap only partially.

In a spectroscopic setting, the purpose of a weak QND measurement is to conditionally reduce population noise, while keeping the reduction of the signal slope $\eta$ due to decoherence low, such that the SNR improves, i.e., $\operatorname{var}_{\text {cond }}\left(\Delta N / \eta N_{\text {at }}\right)<\operatorname{var}\left(\Delta N / N_{\text {at }}\right)$, the Wineland criterion for spin squeezing [23]. To investigate the influence of atomic motion and coupling inhomogeneity, in the following we introduce a framework on how to operate QND measurements under such conditions.

As in the projection noise measurement, ensembles with varying atom numbers are prepared in $|\Psi\rangle$ and probed for $8 \mu \mathrm{s}$ and after a $32 \mu \mathrm{s}$ pause again for another $120 \mu \mathrm{s}$ [see Fig. 1(d)]. As a first step, we investigate the temporal correlations within the acquired single-shot phase signals $\vec{\varphi}(t)$ and develop a quantitative model for their first and second statistical moments to understand the underlying physical processes. In a second step, we then use this model to determine the optimal detection mode functions for this given experimental sequence.

From each single experimental trace we determine $\varphi_{N}:=$ $\varphi_{3}+\varphi_{4}$ according to Eq. (2). To eliminate the influence of atom number fluctuations when loading the MOT, we then subtract from each sample of the time trace $\vec{\varphi}\left(t<t_{\text {flip }}\right)$ its expectation value $\left(\varphi_{N} / 2\right) \vec{m}(t)$ to obtain the fluctuations $\overrightarrow{\delta \varphi}\left(t<t_{\text {flip }}\right):=\vec{\varphi}\left(t<t_{\text {flip }}\right)-\left(\varphi_{N} / 2\right) \vec{m}(t)$ and successively their (symmetric) covariance matrix $C=\left\langle\overrightarrow{\delta \varphi} \cdot \overrightarrow{\delta \varphi} \varphi^{\top}\right\rangle$.

As expected for quantum fluctuations, $\boldsymbol{C}=\boldsymbol{C}_{\mathbf{0}}+\varphi_{N} \boldsymbol{C}_{\mathbf{1}}$ can be decomposed into a constant part $\boldsymbol{C}_{\mathbf{0}}$ and a linear, atom-number-dependent part $\boldsymbol{C}_{\boldsymbol{1}}$. As in the projection noise measurement, we find no quadratic contribution scaling with $\varphi_{N}{ }^{2} \cdot \boldsymbol{C}_{\mathbf{0}}$ describes the measurement noise in the absence of atoms originating from the phase shot noise of the low-intensity probe light; as it represents uncorrelated, constant phase shot noise, it can be approximated as $\boldsymbol{C}_{\mathbf{0}}=\delta \varphi_{\mathrm{sn}}{ }^{2} \cdot \mathbf{1}$ [see lower left-hand part of Fig. 3(a)]. $\boldsymbol{C}_{\mathbf{1}}$, depicted in the upper right of Fig. 3(a), describes atomic quantum fluctuations, such as projection and partition noise. The diagonal elements of $\boldsymbol{C}_{\boldsymbol{1}}$ follow the square of the evolving mean phase shift as $\left[\left(\varphi_{N} / 2\right) \vec{m}(t)\right]^{2} / N_{\text {eff }}$ [see Fig. 3(b). After normalizing rows and columns of $\boldsymbol{C}_{\mathbf{1}}$ by this trace, we obtain the matrix of correlation coefficients (not displayed) which is of Töplitz form with minor diagonals as given in the top inset of Fig. 3(a). In this trace, we observe a rapid oscillatory decay of the correlations to about half their initial value with an oscillation period of $11 \mu \mathrm{s}$ and a similar damping time constant. This can be understood to originate from the radial oscillation of the atoms within the 
(a)
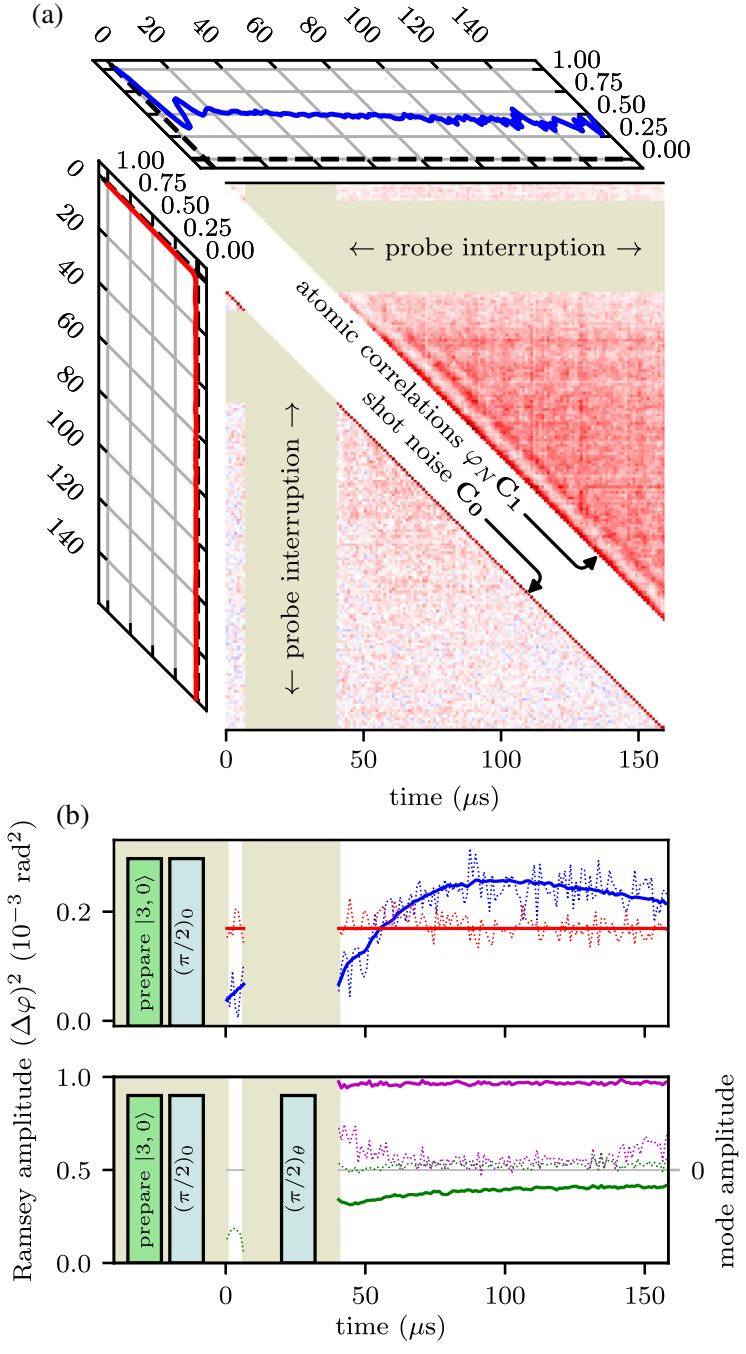

FIG. 3. (a) Constant part $\boldsymbol{C}_{\mathbf{0}}$ (lower left-hand triangle: shot noise) and linear part $\varphi_{N} \boldsymbol{C}_{\mathbf{1}}$ (upper right-hand triangle: atomic correlations) of the symmetric covariance matrix $\boldsymbol{C}=\boldsymbol{C}_{\mathbf{0}}+\varphi_{N} \boldsymbol{C}_{\mathbf{1}}$ for $\varphi_{N}=0.16(1) \mathrm{rad}$ (see text); red (blue) denotes positive (negative) correlations. Shaded areas indicate interruption of the probing. Side panels: Correlation coefficient obtained by averaging over the minor diagonals of the corresponding matrix of correlation coefficients. (b) Upper panel: QND pulse sequence; temporal dependence of the main diagonal entries of $\boldsymbol{C}_{\mathbf{0}}$ (constant part, red dots) and of $\varphi_{N} \boldsymbol{C}_{\mathbf{1}}$ (atom number dependent part, blue dots). Solid blue line, $\left(\varphi_{N} \vec{m}(t) / 2\right)^{2} / N_{\text {eff }}$; solid red line, phase shot noise $\delta \varphi_{\mathrm{sn}}{ }^{2}$. Lower panel: Ramsey-type pulse sequence and temporal evolution of the Ramsey fringe contrast in the absence (solid purple) or presence (solid green) of an $8 \mu$ s probe pulse. Dotted traces denote the corresponding optimal detection mode function.

strongly anharmonic trapping potential: Because of the inhomogeneous probe field, within only half a trap oscillation the probed atomic mode has changed significantly with only minor revivals.

For metrology purposes and spin squeezing, the amount of decoherence caused by a premeasurement pulse [24] is crucial. By inserting a $\pi / 2$-microwave pulse during the $32 \mu$ s probing break, under otherwise unchanged probing conditions, we evaluate the loss of Ramsey fringe contrast $\vec{\eta}(t)$ to determine the "destructiveness" of the first $8 \mu \mathrm{s}$ probe interval [see Fig. 3(c)]. This central characteristic is important for protocols such as QND-measurement-based spin-squeezing and quantum metrology applications as $\vec{\eta}(t)$ is directly proportional to the signal amplitude. The effect of the atomic motion is also visible in this measurement: a slight increase of the fringe contrast is observed with increasing probing time, as atoms affected by the initial decohering light pulse are progressively leaving the probe region and are replaced by more weakly probed ones. In this measurement, due to the duration of the microwave pulse and the imposed $32 \mu$ s delay until probing commences, the damped oscillatory feature seen in the correlation coefficients was not directly accessible and is surmised to have decayed by the time when the Ramsey contrast is probed.

For a temporal detection mode $\vec{q}(t)$, the metrologically relevant phase resolution is given by the square root of the signal-to-noise ratio $\mathrm{SNR} \equiv\left(\vec{q}^{\top} \cdot \vec{s} \cdot \vec{s}^{\top} \cdot \vec{q}\right) /\left(\vec{q}^{\top} \cdot \boldsymbol{C} \cdot \vec{q}\right)$, with $\vec{s}(t)=\varphi_{N} \vec{m}(t) \vec{\eta}(t)$ being the decoherence-affected signal strength and $\boldsymbol{C}$ the covariance matrix. Knowing both $\vec{m}(t) \vec{\eta}(t)$ and $\boldsymbol{C}$, we can directly obtain the optimal temporal probe mode function that maximizes the SNR for both the spin-squeezing premeasurement and the spectroscopic measurement using a matched filter [25] as $\vec{q}_{\text {opt }}(t)=$ $\boldsymbol{C}^{-\mathbf{1}} \cdot \vec{s}(t)$ [see Fig. 3(c)]. However, in this experiment, due to the low optical depth of the present ensemble (od $=8$ for atoms in $|4,0\rangle$ ) and the rapid decay of the correlations, even with the optimal detection mode, using a QND premeasurement we cannot improve spectroscopic resolution compared to a conventional Ramsey sequence.

Several technical measures can push the system deeper into the quantum regime. Utilizing the hyperfine coherence between stretched Zeeman levels, the optical depth for the premeasurement can be increased by a factor of 3 . Working with faster coherent operations and stronger probing, the measurement can outrun the thermal motion induced decay of correlations. Last, but not least, the decay of correlations can be greatly reduced by cooling the atoms into the motional ground state of the lattice sites [26-28].

Given the inherent inhomogeneous coupling between light and atoms in nanoscale traps, it is relevant to discuss the limits posed by quantum mechanics on the performance of such inhomogeneous dispersive atom-light interfaces. As in the case of homogeneous QND coupling, the noisy quantum backaction onto the atomic coherence is mediated by residual spontaneous scattering of probe light. In the inhomogeneous case the change of motional state due to photon recoil leads to additional noise, since the coupling generally depends on the trap level. The probability for leaving the motional ground state in a single scattering event is suppressed by the Lamb-Dicke parameter $\eta^{2}=\omega_{\text {rec }} / \omega_{\text {trap }}$, 
implying that strong enough confinement and low temperature allow us to keep the extra noise far below the inevitable dephasing. We note that engineering the dissipation by channeling a sizable fraction of spontaneous emission into the fiber-guided modes mediates long-range spin-dependent interaction between the atoms. This changes the simple picture and opens a rich field of many-body physics for study $[10,29,30]$.

\section{CONCLUSION}

We present an experimental analysis of atomic quantum noise detection for ensembles trapped in the evanescent field of an optical nanofiber and identify the main challenges to be overcome for truly quantum enhanced applications. Our work paves the way to entanglement generation of a few atoms using solely the light propagating in a nano-optical waveguide interface. As a single nanofiber system is the host of two mesoscopic one-dimensional atomic ensembles, it offers the exciting prospect of a joint QND (Bell) measurement of the two ensembles, a wellidentified goal for EPR entanglement and teleportation protocols based on two-mode squeezing. For these applications an integrated fiber cavity can significantly enhance the effective optical depth of the ensemble [5,31] and improve on the degree of achievable squeezing.

\section{ACKNOWLEDGMENTS}

This work has been supported by the ERC grant INTERFACE (Grant No. ERC-2011-ADG 20110209), the U.S. ARO Grant No. W911NF-11-0235, the EU grant SIQS (Grant No. 600645), and the John Templeton Foundation.

[1] K. Hammerer, A. S. Sørensen, and Eugene S. Polzik, Quantum Interface between Light and Atomic Ensembles, Rev. Mod. Phys. 82, 1041 (2010).

[2] E. Vetsch, D. Reitz, G. Sagué, R. Schmidt, S. T. Dawkins, and A. Rauschenbeutel, Optical Interface Created by LaserCooled Atoms Trapped in the Evanescent Field Surrounding an Optical Nanofiber, Phys. Rev. Lett. 104, 203603 (2010).

[3] A. Goban, K. S. Choi, D. J. Alton, D. Ding, C. Lacroûte, M. Pototschnig, T. Thiele, N. P. Stern, and H. J. Kimble, Demonstration of a State-Insensitive, Compensated Nanofiber Trap, Phys. Rev. Lett. 109, 033603 (2012).

[4] J.-B. Béguin, E. M. Bookjans, S. L. Christensen, H. L. Sørensen, J. H. Müller, E. S. Polzik, and J. Appel, Generation and Detection of a Sub-Poissonian Atom Number Distribution in a One-Dimensional Optical Lattice, Phys. Rev. Lett. 113, 263603 (2014).

[5] S. Kato and T. Aoki, Strong Coupling between a Trapped Single Atom and an All-Fiber Cavity, Phys. Rev. Lett. 115, 093603 (2015).
[6] C. Sayrin, C. Clausen, B. Albrecht, P. Schneeweiss, and A. Rauschenbeutel, Storage of Fiber-Guided Light in a Nanofiber-Trapped Ensemble of Cold Atoms, Optica 2, 353 (2015).

[7] B. Gouraud, D. Maxein, A. Nicolas, O. Morin, and J. Laurat, Demonstration of a Memory for Tightly Guided Light in an Optical Nanofiber, Phys. Rev. Lett. 114, 180503 (2015).

[8] H. L. Sørensen, J.-B. Béguin, K. W. Kluge, I. Iakoupov, A. S. Sørensen, J. H. Müller, E. S. Polzik, and J. Appel, Coherent Backscattering of Light off One-Dimensional Atomic Strings, Phys. Rev. Lett. 117, 133604 (2016).

[9] N. V. Corzo, B. Gouraud, A. Chandra, A. Goban, A. S. Sheremet, D. V. Kupriyanov, and J. Laurat, Large Bragg Reflection from One-Dimensional Chains of Trapped Atoms Near a Nanoscale Waveguide, Phys. Rev. Lett. 117, 133603 (2016).

[10] D. E. Chang, L. Jiang, A. V. Gorshkov, and H. J. Kimble, Cavity QED with Atomic Mirrors, New J. Phys. 14, 063003 (2012).

[11] R. Schmied and P. Treutlein, Tomographic Reconstruction of the Wigner Function on the Bloch Sphere, New J. Phys. 13, 065019 (2011).

[12] R. J. Sewell, M. Napolitano, N. Behbood, G. Colangelo, and M.W. Mitchell, Certified Quantum Non-Demolition Measurement of a Macroscopic Material System, Nat. Photonics 7, 517 (2013).

[13] J. Appel, P. J. Windpassinger, D. Oblak, U. Busk Hoff, N. Kjærgaard, and E. S. Polzik, Mesoscopic Atomic Entanglement for Precision Measurements beyond the Standard Quantum Limit, Proc. Natl. Acad. Sci. U.S.A. 106, 10960 (2009).

[14] X. Qi, B. Q. Baragiola, P. S. Jessen, and I. H. Deutsch, Dispersive Response of Atoms Trapped Near the Surface of an Optical Nanofiber with Applications to Quantum Nondemolition Measurement and Spin Squeezing, Phys. Rev. A 93, 023817 (2016).

[15] D. Reitz, C. Sayrin, R. Mitsch, P. Schneeweiss, and A. Rauschenbeutel, Coherence Properties of NanofiberTrapped Cesium Atoms, Phys. Rev. Lett. 110, 243603 (2013).

[16] The arrow denotes time-dependent functions (column vectors of samples), and we define $\vec{\varphi} \equiv\left(\overrightarrow{\phi_{+}}-\overrightarrow{\phi_{-}} / 2\right)$ with $\overrightarrow{\phi_{ \pm}}$ describing the optical phase shift of the upper (lower) frequency probes.

[17] Caused by $30 \%$ transmission losses, $89 \%$ detector quantum efficiency, $90 \%$ interference visibility, and $20 \%$ equivalent losses due to electronic noise.

[18] R. Mitsch, C. Sayrin, B. Albrecht, P. Schneeweiss, and A. Rauschenbeutel, Quantum State-Controlled Directional Spontaneous Emission of Photons into a Nanophotonic Waveguide, Nat. Commun. 5, 5713 (2014).

[19] C. Sayrin, C. Junge, R. Mitsch, B. Albrecht, D. O'Shea, P. Schneeweiss, J. Volz, and A. Rauschenbeutel, Nanophotonic Optical Isolator Controlled by the Internal State of Cold Atoms, Phys. Rev. X 5, 041036 (2015).

[20] R. McConnell, H. Zhang, J. Hu, S. Cuk, and V. Vuletic, Entanglement with Negative Wigner Function of Almost 3,000 Atoms Heralded by One Photon, Nature (London) 519, 439 (2015). 
[21] $\beta=3.6$ is slightly larger than the factor 3 expected from the $|4\rangle \leftrightarrow\left|5^{\prime}\right\rangle$ Clebsch-Gordon coefficients, as atoms in $|4,4\rangle$ are pulled towards the fiber by the probe, which increases their coupling.

[22] M. H. Schleier-Smith, I. D. Leroux, and V. Vuletić, States of an Ensemble of Two-Level Atoms with Reduced Quantum Uncertainty, Phys. Rev. Lett. 104, 073604 (2010).

[23] D. J. Wineland, J. J. Bollinger, W. M. Itano, F. L. Moore, and D. J. Heinzen, Spin Squeezing and Reduced Quantum Noise in Spectroscopy, Phys. Rev. A 46, R6797 (1992).

[24] A. Louchet-Chauvet, J. Appel, J. J. Renema, D. Oblak, N. Kjaergaard, and E. S. Polzik, Entanglement-Assisted Atomic Clock beyond the Projection Noise Limit, New J. Phys. 12, 065032 (2010).

[25] G. Turin, An Introduction to Matched Filters, IRE Trans. Inf. Theory 6, 311 (1960).

[26] B. Albrecht, Y. Meng, C. Clausen, A. Dareau, P. Schneeweiss, and A. Rauschenbeutel, Fictitious Magnetic-Field Gradients in Optical Microtraps as an Experimental Tool for Interrogating and Manipulating Cold Atoms, Phys. Rev. A 94, 061401 (2016).

[27] C. Østfeldt, J.-B.S. Béguin, F. T. Pedersen, E. S. Polzik, J. H. Müller, and J. Appel, Dipole Force Free Optical Control and Cooling of Nanofiber Trapped Atoms, Opt. Lett. 42, 4315 (2017).

[28] Y. Meng, A. Dareau, P. Schneeweiss, and A. Rauschenbeutel, Near-Ground-State Cooling of Atoms Optically Trapped $300 \mathrm{~nm}$ Away from a Hot Surface, arXiv:1712.05749.

[29] A. González-Tudela, V. Paulisch, D. E. Chang, H. J. Kimble, and J. I. Cirac, Deterministic Generation of Arbitrary Photonic States Assisted by Dissipation, Phys. Rev. Lett. 115, 163603 (2015).

[30] A. Asenjo-Garcia, M. Moreno-Cardoner, A. Albrecht, H. J. Kimble, and D. E. Chang, Exponential Improvement in Photon Storage Fidelities Using Subradiance and "Selective Radiance" in Atomic Arrays, Phys. Rev. X 7, 031024 (2017).

[31] S. K. Ruddell, K. E. Webb, I. Herrera, A. S. Parkins, and M. D. Hoogerland, Collective Strong Coupling of Cold Atoms to an All-Fiber Ring Cavity, Optica 4, 576 (2017). 